Rev. salud pública. 14 sup (2): 46-57, 2012

\title{
Inequidades en salud comunicativa: el caso del aprendizaje inicial de la escritura
}

\section{The initial stages of learning to write: age and socioeconomic differences}

\author{
Rita Flórez-Romero' ${ }^{1}$, Nicolás Arias-Velandia² y Luisa-F. Benítez-Camargo ${ }^{2}$
}

1 Facultad de Medicina,Universidad Nacional de Colombia. Bogotá. rflorezr@unal.edu.co

2 Investigadores Independientes. Bogotá, Colombia.

Recibido 25 Mayo 2011/Enviado para Modificación 8 Agosto 2011/Aceptado 3 Noviembre 2011

\section{RESUMEN}

Objetivos Establecer similitudes y diferencias entre los desempeños de niños en jardines infantiles de estratos socioeconómicos altos (4 y 5 ) y estratos socioeconómicos bajos (1 y 2) en escritura como manifestación de inequidad en la salud comunicativa de los niños preescolares.

Procedimientos Se compararon los rendimientos en las tareas de la Evaluación de Escritura Inicial y Emergente (ESEMER) en edad (3, 4 y 5 años) y estratos socioeconómicos (altos o 4 y 5 , y bajos o 1 y 2) en 223 niños.

Resultados destacados La escritura como notación se correlacionó con la edad de los niños, la escritura como estilo de discurso mostró diferencias entre los estratos altos y bajos, y la escritura como modo de producción se correlacionó con la edad y con el estrato socioeconómico.

Principales conclusiones La escritura como estilo de discurso es más rica en los estratos altos que en los bajos y también varía entre los jardines infantiles de estrato alto. Estas diferencias se deben a inequidad en la salud comunicativa de los niños participantes, reflejada en sus niveles de acceso al conocimiento significativo de la escritura.

Palabras Clave: Desigualdades en la salud, barreras de comunicación, escritura manual, preescolar (fuente: DeCS, BIREME).

\section{ABSTRACT}

Objectives Establishing similarities and differences between the performance of children from higher socioeconomic strata (4 and 5) attending kindergartens and those from low socioeconomic strata (1 and 2) concerning writing, notation, mode of production and style of speech.

Methods A set of initial and emergent writing assessment (IEWA) tasks were compared to evaluate three aspects of knowledge regarding writing with 223 children aged 3, 4 and 5 years of age from four kindergartens dealing with children 
from socioeconomic strata 4 and 5 (high ) and three kindergartens taking children from level 1 and 2 (low) in Bogotá. Performance was compared in terms of age and high and low strata.

Results Writing as notation was correlated with children's age; writing and speaking styles revealed differences between high and low strata and writing as a mode of production was correlated with age and socioeconomic status.

Conclusions Writing as a style of discourse (notation) was richer in higher strata children and varied between kindergartens taking high strata children because of language comprehension being dealt with as a system. Such differences were due to children's unequal access to the significant meaning implicit in writing.

Key Words: Inequality, writing, preschool (source: MeSH, NLM).

$\mathrm{S}$ egún la Organización Mundial de la Salud, el desarrollo del potencial de los niños en la infancia temprana tiene impacto en toda su salud posterior e incide en la reducción de inequidades. Dicha organización recomienda entonces: a. desarrollar amplios programas de calidad para todos los niños, madres y cuidadores en la primera infancia y b. proveer educación primaria y secundaria de calidad para todos los niños (1). En el mismo sentido se considera que las experiencias tempranas pobres llevan a un mayor riesgo de padecer problemas de salud físicos y mentales $(1,2)$.

Lo anterior, también se manifiesta en las idea de la salud como derecho. Al asumirla de esta manera, la salud forma parte también de los derechos sociales y culturales, además de hacer parte de los derechos humanos fundamentales (3). Dentro de este derecho se inscribe la salud y el bienestar comunicativo, que se incrementa con prácticas que lleven a las personas a utilizar el lenguaje en propósitos que están más allá de la satisfacción de necesidades en la experiencia inmediata. A su vez, dichas prácticas, permiten a los niños acumular un acervo que les va a permitir derivar satisfacción de sus experiencias a través de la comunicación, como en el aprendizaje temprano de la escritura (4).

El dominio de competencias alfabéticas como la escritura favorece el acceso de las personas al acervo de conocimientos acumulados por la humanidad y su participación informada $(5,6)$. Para garantizar este derecho, los educadores iniciales y las autoridades e interesados en el desarrollo y el aprendizaje de los pequeños deben conocer los aspectos involucrados en la oralidad, la lectura y la escritura (5), con el fin de dar soporte a intervenciones con beneficio (eficacia) y de costo (eficiencia) en 
esta área (7). La escritura, a diferencia de habilidades del lenguaje oral, necesita de instrucción o enseñanza explícita, o de actividades y materiales planeadas por los adultos alrededor $(2,5,9)$. La escritura es también la actividad de componer mensajes utilizando el lenguaje escrito (que incluye todo el proceso comprendido en planear, transcribir, revisar y editar el texto escrito, 10), y esta actividad tiene dos dimensiones: la epistémica (transformación del conocimiento durante la actividad de escribir) y la comunicativa (precisión de un mensaje y de su forma para hacerlo claro, directo y ameno para un destinatario) $(9,10)$. La actividad de escribir es también una herramienta metacognitiva o de revisión, control y regulación del conocimiento que ha adquirido una persona $(5,11,12)$.

Lo anterior, muestra que el aprendizaje de la escritura demanda al niño plantear hipótesis que le permitan avanzar en su comprensión de diferentes aspectos y niveles de la actividad de escribir (13). Se entiende que tanto la escritura como otros conocimientos relacionados con el alfabetismo comienzan su aprendizaje antes de la enseñanza formal de los mismos en el primer grado de la escuela primaria $(14,15)$. En los diferentes entornos sociales los niños tienen diferentes oportunidades de aprendizaje de la escritura, con lo cual se evidencia que este factor de oportunidad y de acceso al bienestar $(5,15,16)$. Dichos conocimientos se fortalecen en los niños a partir de la implementación de programas o actividades de instrucción que les den oportunidades de avanzar en su aprendizaje de la escritura (17) a través de prácticas agradables para los niños pero también que incluyan oportunidades de revisar y corregir la elaboración de textos propios y de otros con ayuda del adulto, con instrucciones explícitas sobre distintos aspectos de la escritura $(10,18-20)$.

El proceso por el cual los niños llegan al aprendizaje de la escritura consiste en la reconstrucción de diferentes pasos que hace el niño, junto con las oportunidades del entorno y de los adultos que le enseñan, de diferentes aspectos y niveles que constituyen esta actividad (9). Dicha reconstrucción se da en tres niveles de análisis en el aprendizaje de la escritura de acuerdo con Tolchinsky (21): la escritura como notación, la escritura como modo de producción y la escritura como diferenciación de géneros discursivos. El aprendizaje de la escritura como notación se refiere al desarrollo de la comprensión del principio alfabético o correspondencia entre caracteres escritos y sonidos de la lengua. El aprendizaje de la escritura como modo de producción se refiere a la comprensión lograda por el niño de los elementos de organización que debe tener un texto escrito, como la organización de la información, la organización 
de oraciones, la acomodación al destinatario o personas que lo va leer y la selección de palabras, frases y formas gramaticales de los enunciados que se usan en su organización y composición. El aprendizaje de escritura como diferenciación de géneros discursivos se refiere a la comprensión de diferentes géneros que responden a distintos propósitos comunicativos en la escritura, como manuscritos, periódicos, libros, cartas, y otros. En esta perspectiva, se hacen al niño demandas de organización de la información y de conocimiento del código alfabético, sin privilegiar uno solo de ellos en detrimento de otro (22).

El objetivo de este estudio es observar similitudes y diferencias en el aprendizaje de la escritura como notación, como modo de producción y como diferenciación de géneros discursivos en niños de jardines infantiles con diferente estratificación socioeconómica que habitualmente muestra diferencias importantes en las oportunidades de aprendizaje alfabético que dan a sus niños.

\section{MÉTODO}

\section{Sujetos}

223 niños y niñas de 3, 4 y 5 años de edad inscritos en siete jardines infantiles y colegios de la ciudad de Bogotá D.C.143 niños (64,2\%), son de jardines infantiles de estrato socioeconómico 4 - 5 y 80 (35,8\%), de jardines infantiles de estrato socioeconómico1-2. De todos los participantes, 6 son de nivel párvulos (2,7 \%), 91 de nivel prejardín (40,8\%), 51 de nivel jardín $(22,9 \%), 20$ de prekinder (9\%), 17 de kínder (7,6 \%), y 38 de transición (17\%). El promedio de edad de estos niños es de 51,62 meses (4 años y 4 meses), con desviación estándar de 10,612 meses.

Diseño

Este es un estudio correlacional que compara el logro en el aprendizaje de la escritura como notación, como modo de producción y como estilo de discurso en niños de estrato socioeconómico medio-alto (4-5) y de estrato bajo (1-2) en un solo corte transversal.

\section{Instrumento}

Se aplicó la Evaluación de Escritura Inicial y Emergente (ESEMER), elaborada por Rita Flórez Romero, Nicolás Arias Velandia y Diana Paola Gómez Muñoz, que tiene cuatro partes: preguntas sobre las creencias de 
los niños sobre la escritura y lectura; conocimientos de los niños sobre la escritura como notación; conocimientos sobre la escritura como modo de producción y conocimientos sobre la escritura como género discursivo. Las respuestas de la primera sección son audiograbadas, a las tres siguientes se consignaron en hojas de respuesta y las producciones de los niños están en hojas adjuntas.

Procedimientos

Se aplicó la Evaluación de Escritura Inicial y Emergente (ESEMER) a los niños en el orden señalado en dicha evaluación. En un recinto fuera de los salones de cada jardín infantil, cada evaluador llevó de a un niño y realizó aplicación individual del instrumento ESEMER. Primero, se les explicó a los niños que se les realizarían algunas preguntas y que se les pediría que realizaran las tareas solicitadas. Si el niño mostraba disposición para continuar, se seguía con las preguntas y con los ítems de la evaluación en el orden establecido. Se continuaba la evaluación con los niños hasta el final, salvo en tres situaciones: cuando el niño mostrara cansancio o fatiga, cuando mostrara indisposición para seguir respondiendo a la evaluación, o cuando diera muestras repetidas de no comprender las preguntas.

Estadísticas y análisis

Este estudio utilizó dos cálculos estadísticos para el análisis de la información obtenida: la prueba t de Student de comparación de muestras independientes y el coeficiente de correlación de Spearman. La prueba t de Student de comparación de muestras independientes compara la distancia entre las medias de los rendimientos de los niños de ambos grupos de estratificación socioeconómicos (medio-alto y bajo). Se consideran significativas las diferencias si la diferencia de medias resultante está por encima del intervalo de confianza de $95 \%$. El coeficiente de correlación de Spearman sirve para establecer si hay covariación o variación en el mismo sentido entre los rendimientos de los niños y su edad. La correlación se considera alta o significativa si es mayor a $0.4 \mathrm{y}$ si el nivel de significancia es menor de 0.05. Los datos obtenidos se procesaron en una base de datos en el paquete estadístico informático Statistic Packet for Social SciencesSPSS 17 ®. 


\section{RESULTADOS}

Los conocimientos de la escritura como notación tienen correlación alta con la edad del niño en meses, (Pearson=0,690; Sig.=0,000; N=222). La edad del niño también tiene correlaciones significativas entre bajas y moderadas con la escritura de elementos presentes en una imagen, (Pearson=0,379; Sig. $=0,000 ; \mathrm{N}=221)$; con escribir la descripción de lo que sucede en una imagen, (Pearson=0,401; Sig. $=0,000 ; \mathrm{N}=222$ ); y con la escritura como sistema de producción (Pearson=0,451; Sig. $=0,000 ; \mathrm{N}=220$ ); y con la escritura de todas las letras que se conocen (Tau-b Kendall=4,000; Sig. $=0,000 ; \mathrm{N}=222$ ). Los análisis de varianza (ANOVA) mostraron que la edad muestra efectos significativos en las creencias del niño sobre la lectura y la escritura y en las creencias de los niños sobre la lectura y la escritura (Tabla 1).

Tabla 1. Análisis de varianza (ANOVA) de los efectos de la edad sobre la escritura como notación y sobre las creencias de los niños

\begin{tabular}{lcccccc}
\hline & & $\begin{array}{c}\text { Suma de } \\
\text { cuadrados }\end{array}$ & gl & $\begin{array}{c}\text { Media } \\
\text { cuadrática }\end{array}$ & $F$ & Sig. \\
\hline Total escritura como & Inter-grupos & 25.802 & 38 & 0,679 & 7,537 & 0,000 \\
notación & Intra-grupos & 16.487 & 183 & 0,090 & & \\
Total creencias de los & Inter-grupos & 15.437 & 38 & .406 & 1,643 & 0,017 \\
niños sobre la lectura & Intra-grupos & 45.257 & 183 & .247 & & \\
y la escritura & Total & 60.695 & 221 & & & \\
\hline
\end{tabular}

Los análisis de variación no paramétricos de Kruskall - Wallis también mostraron relaciones significativas entre la edad y aspectos puntuales del conocimiento del niño sobre la escritura como notación y en los conocimientos del niño sobre la escritura como sistema de producción (Tabla 2).

Tabla 2. Estadísticos de contraste a, b del cálculo no paramétrico de KruskallWallis sobre la relación entre la edad de los niños y distintos niveles de análisis en la escritura inicial

\begin{tabular}{lccccc}
\hline & $\begin{array}{c}\text { Escribe } \\
\text { todas las } \\
\text { letras que } \\
\text { conoces }\end{array}$ & $\begin{array}{c}\text { Escribe el } \\
\text { nombre de } \\
\text { las cosas que } \\
\text { aparecen acá }\end{array}$ & $\begin{array}{c}\text { Escribe } \\
\text { lo que } \\
\text { pasa acá } \\
\text { (imagen) }\end{array}$ & $\begin{array}{c}\text { Escritura } \\
\text { como } \\
\text { sistema de } \\
\text { producción }\end{array}$ & $\begin{array}{c}\text { Escritura como } \\
\text { diferenciación } \\
\text { de géneros } \\
\text { discursivos }\end{array}$ \\
Chi-cuadrado & 103.152 & 71.697 & 90.800 & 80.602 & 48.040 \\
Gl & 38 & 38 & 38 & 38 & 38 \\
Sig. asintót. & .000 & .001 & .000 & .000 & .127 \\
\hline
\end{tabular}

a. Prueba de Kruskal-Wallis b. Variable de agrupación: Edad del niño 
El nivel socioeconómico correlacionó significativamente con escritura como diferenciación de géneros discursivos, (Rho Spearman=0,539; Sig. $=0,000 ; \mathrm{N}=223$ ) y con las creencias sobre la lectura y la escritura, (Rho Spearman=0,187; Sig.=,005; N 223); y con la escritura como sistema de producción (Rho Spearman=0,186; Sig. $=0,006 ; \mathrm{N}=221$ ).

La comparación entre estratos por medio de la prueba estadística t de Student y la prueba no paramétrica U de Mann-Whitney (Tabla 3) para muestras independientes mostró diferencias significativas en favor de los niños de nivel socioeconómico medio-alto en escritura como sistema de producción y en escritura como diferenciación de géneros discursivos.

Hubo un efecto importante del tipo de colegio en las instituciones de nivel socioeconómico alto en los conocimientos de los niños sobre la escritura como notación (Tabla 4), con mayor rendimiento en el Jardín Infantil 1, seguido de Jardín Infantil 2, Jardín Infantil 3 y Jardín Infantil 4 (que muestra rendimientos significativamente menores que todos los otros) (Anexo 1).

Tabla 3. Prueba no paramétrica $U$ de Mann-Whitney de diferencias del desempeño por estrato medio-alto o bajo en los tres niveles de aprendizaje inicial de la escritura en ESEMER

\begin{tabular}{lccccc}
\hline \multicolumn{1}{c}{ Prueba } & $\begin{array}{c}\text { Escribe } \\
\text { todas las } \\
\text { letras que } \\
\text { conoces }\end{array}$ & $\begin{array}{c}\text { Escribe el } \\
\text { nombre de } \\
\text { las cosas que } \\
\text { aparecen acá }\end{array}$ & $\begin{array}{c}\text { Escribe } \\
\text { lo que } \\
\text { pasa acá } \\
\text { (imagen) }\end{array}$ & $\begin{array}{c}\text { Escritura } \\
\text { como } \\
\text { sistema de } \\
\text { producción }\end{array}$ & $\begin{array}{c}\text { Diferenciación } \\
\text { entre tipos de } \\
\text { texto }\end{array}$ \\
\hline $\begin{array}{l}\text { U de Mann- } \\
\text { Whitney }\end{array}$ & 5313000 & 5609500 & 5686500 & 4619.000 & 2362000 \\
W de Wilcoxon & 15609000 & 8769500 & 8926500 & 7779000 & 5602000 \\
Z & -1.325 & -.134 & -.132 & -2.753 & -8.031 \\
$\begin{array}{l}\text { Sig. asintót. } \\
\text { (bilateral) }\end{array}$ & .185 & .893 & .895 & .006 & .000 \\
\hline
\end{tabular}

Tabla 4. Análisis de Varianza (ANOVA) del efecto del jardín infantil en estrato medio-alto sobre los conocimientos de los niños de la escritura como notación

\begin{tabular}{ccccccc}
\hline \multicolumn{2}{c}{ Item } & $\begin{array}{c}\text { Suma de } \\
\text { cuadrados }\end{array}$ & Gl & $\begin{array}{c}\text { Media } \\
\text { cuadrática }\end{array}$ & F & Sig. \\
\hline \multirow{2}{*}{$\begin{array}{c}\text { Total escritura } \\
\text { como notación }\end{array}$} & Inter-grupos & 11497 & 3 & 3832 & 26826 & .000 \\
& $\begin{array}{c}\text { Intra-grupos } \\
\text { Total }\end{array}$ & 19857 & 139 & .143 & & \\
& 31354 & 142 & & & \\
\hline
\end{tabular}




\section{DISCUSIÓN}

Este estudio muestra relaciones de la edad de los niños en meses con la escritura como notación. Esto puede ser explicado por la diversidad de entornos con diferente riqueza en su práctica a los que los niños están expuestos en alguna medida, de los cuales pueden abstraer que hay diferentes signos escritos que conforman un código y que dicho código tiene un significado en el mundo de los adultos $(9,10,13,14,15)$.

De otra parte, estudios como los de Flórez Romero, Torrado Pacheco y Mesa (23), y el de Flórez Romero y colaboradores (12) muestran que ciertos aspectos de organización del lenguaje como sistema tienden a variar poco con factores del entorno y un poco más en función de la edad. Esto se ve corroborado por hallazgos que muestran que a pesar de la diferencia de prácticas, aspectos como la morfosintaxis o el conocimiento de caracteres se ven poco afectados por la diferencia de prácticas de educación inicial que los niños tienen $(13,14,23,24)$.

El segundo hallazgo en este estudio nos plantea un efecto muy importante del estrato socioeconómico sobre la escritura como producción y sobre la escritura como conjunto de géneros discursivos. Estudios previos han hallado también que el nivel socioeconómico tiene una estrecha relación con el crecimiento de conocimientos alfabéticos en el niño $(14,25)$. Los entornos del hogar de los niños pueden diferir mucho en ese grado de riqueza de prácticas que promuevan los conocimientos iniciales en lectura y escritura en los niños, pero a pesar de ello, un soporte del sistema educativo que esté bien diseñado en sus prácticas podría aprovechar las inquietudes del niño en las prácticas pedagógicas para hacer que lleguen al aprendizaje alfabético aún superando estas diferencias socioeconómicas $(2,14,15)$.

Por otra parte, también se ha planteado que desde temprano los niños tienen cierta "sensibilidad" para detectar las diferencias entre tipos de texto según su forma y en el tipo de intención comunicativa que suelen expresar $(17,18,22,26)$. Las diferencias en los dos grupos de niveles socioeconómicos sugieren que este aspecto debe fortalecerse en las prácticas de los programas para la primera infancia $(5,14,22,27,28,29)$.

También resulta interesante constatar que la escritura como sistema de producción hay efecto tanto de edad del niño como del estrato 
socioeconómico. Esto indica que en este aspecto los factores internos y externos del desarrollo infantil suelen coordinarse mutuamente y apoyar el crecimiento de otras capacidades durante la primera infancia (2). La evolución de los conocimientos linguí́sticos avanzados que exige la articulación entre el lenguaje oral (más obediente a pautas endógenas del desarrollo) y el lenguaje escrito (más dependiente de pautas del entorno) como se pone de manifiesto en estas capacidades $(24,29,30)$.

De este estudio puede concluirse que

La escritura como notación tiende a variar en relación con la edad y por ello parece depender más de factores de evolución interna en el desarrollo del niño, apoyados por la complejización en el conocimiento del lenguaje como sistema.

La escritura como conjunto de diferentes géneros discursivos se dan varía en función de la riqueza alfabética del entorno de los niños. El niño expuesto a estas prácticas mejorará su aprendizaje inicial de la escritura. $\mathrm{Si}$ este soporte falla, se generan situaciones de inequidad porque estas diferencias suelen darse entre los niños de familias de estratos medios y altos, y los de estratos bajos.

La escritura como sistema de producción tiene de la edad del niño y del estrato socioeconómico de su jardín infantil, al parecer porque en este tipo de conocimiento se articulan conocimientos del lenguaje oral que en sus aspectos básicos parecen tener una pauta más fuerte de aspectos endógenos en el desarrollo y conocimientos del lenguaje escrito que generalmente tiene una influencia más fuerte la oferta de actividades y contextos alfabéticos

Agradecimientos: Los autores de este trabajo agradecen la colaboración en el proceso y finalización del presente estudio a los Doctores Carlos Julio Pacheco Consuegra y Carlos Alberto Agudelo, Decanos de la Facultad de Medicina de la Universidad Nacional de Colombia, y a Martha Janeth Mojica, jefe de la Oficina de Investigación de la misma Facultad, por el apoyo en la administración de recursos financieros de COLCIENCIAS para el proyecto de investigación que soportó este estudio. También se agradece la colaboración de las profesoras Clemencia Restrepo Arias, Directora del Departamento de Comunicación Humana y de la profesora Luisa Fernanda Ángel Gordillo, Coordinadora Curricular de la Carrera de Fonoaudiología (adscrita al mismo departamento), por facilitarnos medios, infraestructura y espacios de trabajo con estudiantes de pregrado. Se reconoce 
también acá el excelente trabajo como asistentes y auxiliares de investigación de las fonoaudiólogas Angélica María Ballén Ávila, Mayerly Leandra Vega González, Paola Alejandra Espinosa Reyes y Diana Guadalupe Tamayo Medina y de la psicóloga y educadora Magda Inés Medina Manrique. En el mismo sentido, se agradece la retroalimentación en temas de educación inicial a Rosa Julia Guzmán Rodríguez, profesora de la Facultad de Educación de la Universidad de La Sabana. También se agradece especialmente a los jardines infantiles participantes, a sus niños en niveles de educación inicial y a sus padres.

Conflicto de intereses: Este estudio se deriva de otro realizado con el apoyo financiero del Departamento Administrativo de Ciencia, Tecnología e Innovación COLCIENCIAS en la Convocatoria Nacional para la Financiación de Proyectos de Investigación 2008-Modalidad Recuperación Contingente. El estudio financiado es el proyecto de investigación Promoción de la lectura inicial y prevención de las dificultades en comprensión de lectura PROLECIN Segunda fase, a cargo de la autora principal y realizado en el Departamento de Comunicación Humana, Facultad de Medicina, Universidad Nacional de Colombia.

\section{REFERENCIAS}

1. World Health Organization. Social determinants of health. Closing the gap in a generation how? Febrero de 2011. [Internet]. Disponible en: http://www.who.int/ social_determinants/thecommission/finalreport/closethegap_how/en/index1.html. Consultado febrero 2011.

2. Mustard F. Desarrollo infantil inicial y política pública. En: FPAA, eds. Movilización por la Primera Infancia 2o Foro Internacional Memorias, Bogotá: Fondo de acción Ambiental y La Niñez FPAA, Instituto Colombiano de Bienestar Familiar; 2006.p.41-47.

3. Gaviria-Díaz C. El derecho a la salud en el derecho constitucional colombiano. En: Franco S, ed., La Salud Pública hoy. Enfoques y dilemas contemporáneos en salud pública, Bogotá: Universidad Nacional de Colombia; 2002. p.305-314.

4. Cuervo C. La profesión de fonoaudiología: Colombia en perspectiva internacional. Bogotá: Universidad Nacional de Colombia; 1998.

5. Flórez-Romero R, Arias-Velandia N, Cuervo-Echeverri CL. La experiencia del alfabetismo emergente y otras maneras de ver la lectura y la escritura en la educación inicial. En: IDEP edts. Memorias VI Congreso Internacional de Investigación en Educación y Pedagogía, agosto de 2006. Bogotá: Instituto para la Investigación Educativa y el Desarrollo Pedagógico IDEP; 2007.p. 256-262

6. Olson D. El mundo sobre el papel. Barcelona: Gedisa; 1994.

7. Romero-Rey T. Proceso de Formulación de Política de Primera Infancia. En:FPPA eds. Movilización por la Primera Infancia 2o Foro Internacional Memorias, Bogotá: Fondo de acción Ambiental y La Niñez FPAA, Instituto Colombiano de Bienestar Familiar; 2006.p. 29-1.

8. Ferreiro E. La adquisición de los objetos culturales: el caso particular de la lengua escrita. En E. Ferreriro, ed., Alfabetización de niños y adultos. Textos Escogidos, Pátzcuaro: CREFAL; 2007.p. 399-409.

9. Kamhi A, Catts HW. Language and Reading disabilities. Needham Heights, NJ: Prentice Hall; 1999. 
10. Flórez-Romero R; Cuervo-Echeverri CL. El regalo de la escritura. Cómo aprender a escribir. Bogotá: Universidad Nacional de Colombia; 2005.

11. Hurtado-Vergara RD. Factores a considerar en la construcción de una didáctica de la lectura y la escritura en la infancia. En: Programa Palabrario, ed, Lecturas complementarias para maestros. Leer y escribir con niños y niñas, Bogotá: Fundalectura y Fundación Corona.2007; p.139-153.

12. Flórez-Romero R, Torrado-Pacheco MC, Arévalo-Rodríguez I, Mesa - Guechá C, Mondragón-Bohórquez S, Pérez-Vanegas C. Habilidades metalingüísticas, operaciones metacognitivas y su relación con los niveles de competencia en lectura y escritura: un estudio exploratorio. Forma y Función. 2005; 18:15-44.

13. Ferreiro E, Teberosky-Coronado A. Los sistemas de escritura en el niño. Buenos Aires, México: Siglo XXI; 1993.

14. Flórez-Romero R, Restrepo MA, Schwanenflugel P. Promoción del alfabetismo inicial y prevención de las dificultades en la lectura: una experiencia pedagógica en el aula de preescolar. Avances en Psicología Latinoamericana. 2009; 27(1):79-96.

15. Ferreiro E. Acerca de las no previstas pero lamentables consecuencias de pensar solo en la lectura y olvidar la escritura cuando se pretende formar al lector. Lecturas Sobre Lecturas. 2002; 1:31-37.

16. Garrido F. Estudio versus lectura. Lecturas Sobre Lecturas. 2002; 1:39-61.

17. Pritchard RJ, Honeycutt FL. Best practices in implementing a process approach to teaching writing. In: Graham C, MacArthur y J Fitzgerald Eds. Best Practices in Wrinting Instruction, New York: Guilford Press; 2007.p. 28-49.

18. Bialystok E, Luk G, Kwan E. Bilingualism, biliteracy, and learning to read: interactions among languages and writing systems. Scientific Studies of Reading. 2005; 9(1):43-61.

19. Vlachos F, Bonoti F. Explaining age and sex differences children's handwriting: a neurobiological approach. European Journal of Developmental Psychology.2006; 3(2):113-126.

20. Halpern DF, Benbow CP, Geary DC, Gur RC, Hyde JS, Gernsbacher MA. The Science of Sex Differences in Science and Mathematics. Psychological Science in the Public Interest. 2007; 8(1):1-51.

21. Tolchinsky L. The Emergence of Writing. In: MacArthur C, Graham S y Fitzgerald J, eds., Handbook of Writing Research, New York: Guilford Press; 2006.p. 83-95.

22. Flórez-Romero R, Torrado-Pacheco MC, Arias-Velandia N. Leer en familia: horizonte conceptual. En: Fundalectura, ed., Leer en Familia en Colombia. Reporte de Investigación y Experiencias, Bogotá: Fundalectura; 2006.p.19-49.

23. Flórez-Romero R, Torrado-Pacheco MC, Mesa C. Emergencia de las capacidades metalingüísticas. Rev Latin Psicol. 2006; 38(3): 457-475.

24. Karmiloff K, Karmiloff-Smith A. Hacia el lenguaje. Del feto al adolescente. Madrid: Morata; 2005.

25. Flórez-Romero R, Arias-Velandia N. Evaluación de conocimientos previos del aprendizaje inicial de la lectura. Magis, Revista Internacional de Investigación en Educación. 2010; 2(4): 329-344.

26. Laboratorio Latinoamericano de Evaluación de la Calidad de la Educación LLECE Organización de las Naciones Unidas para la Educación, la Ciencia y la Cultura UNESCO. Segundo Estudio Regional Comparativo y Explicativo SERCE. Aportes para la enseñanza de la lectura. Santiago, Chile: Laboratorio Latinoamericano de Evaluación de la Calidad de la Educación LLECE-Organización de las Naciones Unidas para la Educación, la Ciencia y la Cultura UNESCO; 2009.

27. Ferreiro E. Cultura escrita y educación. México: Fondo de Cultura Económica; 1999. Orozco-Hormaza BC, Correa-Restrepo M. Leer, una condición para escribir. Lenguaje y Escuela. 2002; 1:79-98. 
28. Guzmán-Rodríguez RJ, Ghitis T, Ruiz C. Uso de la tecnología en la alfabetización de niños con déficit cognitivo leve. Imágenes de Investigación. 2010; 1:25-31.

29. Flórez-Romero R, Arias-Velandia N, Castro-Martínez JA. Construyendo puentes: lectura y escritura en la educación inicial. En: Fundalectura, ed., ¡Los Niños son un cuento! Lectura en la primera infancia. Memorias 9ํㅡㄹ Congreso Nacional de Lectura. Bogotá: Fundalectura; 2010. p. 67-87. 